\title{
COMUNICACIONES BREVES
}

$72{ }^{1}$ Carmen María Sarabia Cobo

'Aroa Delgado Uría

${ }^{2}$ Cristina Castanedo Pfeiffer

'Profesora Asociada del Área de Enfermería Geriátrica

y Gerontológica. Subdirectora de Enfermería de Atención

Primaria. Servicio Cántabro de Salud.

${ }^{2}$ Profesora Titular del Área de Enfermería Geriátrica

y Gerontológica. E.U.E. Casa de Salud Valdecilla. Universidad

de Cantabria.

\section{Correspondencia:}

Carmen María Sarabia Cobo

EUE Casa de Salud Valdecilla

Avda. Valdecilla, s/n

39011 Santander

E-mail: carmen.sarabia@unican.es
A vueltas con la jubilación

73

\section{Return to your retirement}

\section{RESUMEN}

La edad de jubilación es considerada la barrera para determinar el inicio de la vejez. En los últimos años estamos asistiendo a un cambio político y social respecto a la edad idónea para jubilarse. Un repaso de los últimos estudios sobre el impacto de la jubilación en las personas, puede permitirnos a los profesionales adaptar estrategias adecuadas para hacer frente a estos cambios. En el presente artículo se lleva a cabo una revisión de los últimos estudios e hipótesis que relacionan la jubilación y el funcionamiento cognitivo y el impacto en la calidad de vida.

\section{PALABRAS CLAVE}

Envejecimiento, jubilación, funciones cognitivas.

\begin{abstract}
SUMMARY
The retirement age is considered the gate to determine the onset of old age. In recent years we are witnessing a political and social change regarding the appropriate age for retirement. A review of recent studies on the impact of retirement on people can afford to professionals adapt appropriate strategies to address these changes. This article provides a review of recent studies and hypotheses relating retirement and cognitive functioning and impact on quality of life.
\end{abstract}

\section{KEY WORDS}

Aging, retirement, cognitive functions.

\section{INTRODUCCIÓN}

La edad de jubilación es considerada la barrera para determinar el inicio de la vejez. La diferencia de edades en la jubilación a lo largo del tiempo hace plantearse la laxitud de este concepto, más sujeto a las políticas sociales que al propio concepto de vejez.

Por ejemplo, en España, hasta el año 1986 la edad para jubilarse estaba fijada a los 70 años, para después ser rebajada a los 65 de la actualidad.

En los últimos años estamos asistiendo a un cambio político y social respecto a la edad idónea para jubilarse. Desde la posibilidad de hacerlo a los 60 años, hasta los últimos proyectos políticos internacionales, que postulan retrasarla de los 65 a los 68 años.

Está claro que la jubilación supone un impacto en la persona; de hecho, es considerada un punto de inflexión en la vida de las personas porque algo cambia: las rutinas, la forma de relacionarse con otros, la capacidad económica, etc.
Un repaso de los últimos estudios sobre el impacto de la jubilación en las personas puede permitirnos a los profesionales adaptar las estrategias adecuadas para hacer frente a estos cambios.

El objetivo del presente artículo es hacer un breve repaso de las conclusiones más relevantes de los últimos estudios y teorías sobre el impacto de la jubilación sobre los aspectos psicológicos y de la calidad de vida.

Hay estudios que indican que el mantenimiento de una actividad laboral intelectual mejora el funcionamiento cognitivo de las personas y mantenerse activo intelectualmente aumenta la flexibilidad de las funciones cognitivas (1).

Otros estudios indican que, incluso en la vejez, la realización de tareas complejas tiene un efecto positivo sobre los procesos cognitivos (1) y que mantenerse activo intelectualmente favorece un envejecimiento exitoso (2). 
Asimismo, otros estudios recientes (3) han demostrado que permanecer voluntariamente en el trabajo a una edad avanzada contribuye a mantener un estado de salud y una vida activa.

Otro factor positivamente relacionado con el hecho de seguir trabajando es la satisfacción derivada de la propia actividad profesional (es decir, las posibilidades para el desarrollo y la influencia en el trabajo, la responsabilidad, un trabajo significativo, y la organización del tiempo de trabajo) (4).

\section{TEORÍAS SOBRE LA JUBILACIÓN}

Si atendemos a las teorías más manejadas en el campo de la sociología y la psicología, destacaremos tres en concreto.

Uno de los principales factores que inciden en los efectos de la jubilación es el tipo de actividad profesional que se mantuvo hasta la misma. Este aspecto fue investigado por Phillipson (1987), que concluyó: "es en la transición de la jubilación cuando el individuo hace un uso de los recursos que él o ella ha desarrollado durante la primera mitad de las fases de la vida. En este sentido, la transición no es un paso de una vieja a una nueva vida... sino que más bien es la resolución final entre las ventajas y los inconvenientes adjuntos a la clase social y las posiciones"(5) Phillipson ha estudiado el periodo de la jubilación en tres grupos ocupacionales diferentes: mineros, trabajadores de fábricas de automóviles y arquitectos. Sus resultados mostraron que la jubilación fue más fácil para los arquitectos porque podían reorientar sus habilidades (pintura, carpintería, elaboración de modelos) en el tiempo libre que el periodo de jubilación suponía. Para los trabajadores del automóvil la situación era más problemática debido a que la brecha que se abría entre el trabajo y la jubilación era mayor. Para los mineros, la jubilación era muy diferente: en la comunidad minera tradicional también hay un lugar para los jubilados, por lo que la transición no fue una carga individual, sino que se limita a un fenómeno colectivo. Para los mineros, la jubilación significa la consolidación de un nivel actual de actividad, para los arquitectos la expansión y la

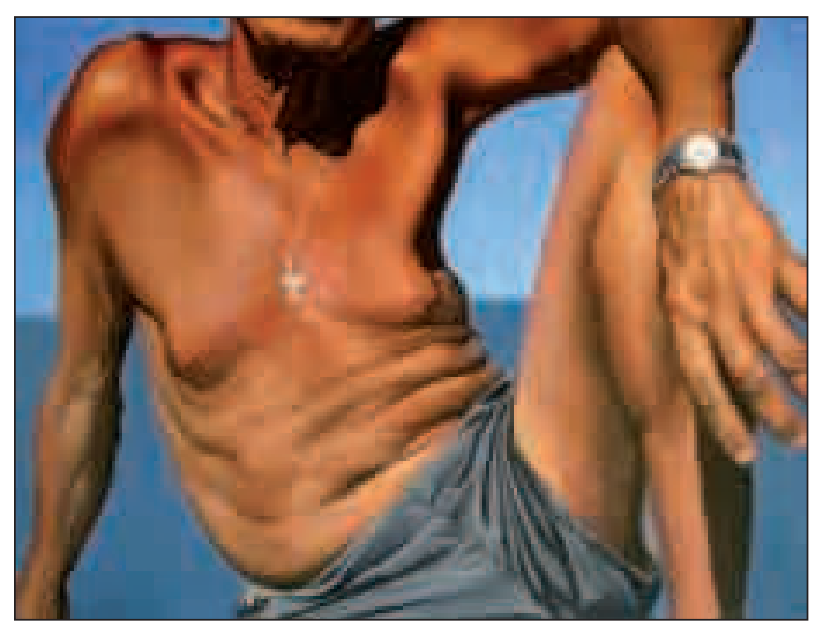

Geografia del tiempo (Daniel García). diversificación de la actividad, y para muchos trabajado- 73 res del automóvil, la pérdida de la actividad.

Un segundo factor a considerar es el tiempo de inactividad desde la jubilación. Una interpretación teórica de los efectos negativos de la jubilación sobre los recursos cognitivos se basa en el principio "úsalo o piérdelo", conocido como la hipótesis del desuso, que postula la idea de que hay diferencias cualitativas, ya sea en la estructura o en el proceso de cognición con el paso de los años. Según esta hipótesis, el uso intensivo y continuo de las habilidades cognitivas básicas durante la edad adulta impide que dichas capacidades desciendan con el paso del tiempo (para una revisión, véase Marquié, 1998) (6). Trabajos recientes en neurociencia apoyan esta hipótesis y demuestran que la plasticidad del cerebro adulto resulta ser mayor de lo esperado $(7,8)$. Un ejemplo se encuentra en el estudio sobre los conductores de taxis de Maguire, Gadian, Johnsrude, Bueno, Ashburner y cols. (9). Este estudio mostró que los taxistas presentaban un hipocampo más grande que los participantes control, lo que sugiere que la función de mantener activa esta estructura, para la orientación, aumentó su volumen. Por otra parte, los conductores más experimentados y de mayor edad presentaban una mayor diferencia respecto a los demás. Sin embargo, aunque estos datos se repiten en estudios similares, siempre se trata de grupos con una experiencia laboral dilatada y muy específica. Por ejemplo, resultados similares, de disminución de las capacidades espaciales asociadas a la edad, se han encontrado en otros grupos con distinta experiencia, incluidos los arquitectos (10). Paralelamente se han encontrado relaciones entre la edad y la memoria musical, independientemente de la experiencia individual (11). Una limitación importante de esta hipótesis es que no se han considerado los afectos y los aspectos motivacionales y el mantenimiento de las funciones cognitivas se atribuye exclusivamente a la experiencia y/o la formación.

Un enfoque más amplio sobre los efectos de la jubilación es el ofrecido por la hipótesis que postula que las personas puedan desarrollar recursos personales que les ayuden a resistir los cambios cognitivos que se producen en el envejecimiento $(12,13)$. Desde este punto de vista, el éxito en el envejecimiento depende de la magnitud de los problemas fisiológicos, cognitivos, de motivación y los recursos sociales disponibles para el individuo.

\section{CONCLUSIONES}

Podemos concluir, por tanto, que la mayoría de estudios y teorías postulan que, con el aumento de la esperanza de vida y de la calidad de vida, es necesario retrasar la edad de inicio de la vejez.

Y que este hecho debe tener implicaciones también en la edad de jubilación. Más allá de las necesidades políticas y sociales que implica la jubilación, debería abrirse un debate constructivo dirigido a validar también el concepto de vejez saludable y sus connotaciones en la calidad de vida $\mathrm{y}$ en las funciones cognitivas a determinada edad. Tal 
74 vez determinadas profesiones requieran, por sus características, un retraso en la edad de jubilación, así como otras que pueden requerir un adelanto.

Tal vez sea necesario, desde el campo de la Geriatría, desligarse de alguna manera de la marca que registra el inicio de la vejez en cuanto a la edad obligatoria de jubilación, porque estamos asistiendo a una clara independencia entre ambas.
La jubilación supone, en la mayoría de casos, mayores 75 desventajas que ventajas a particularizar. Desde hace tiempo sabemos la importancia de desarrollar estrategias para adaptarse a la etapa de jubilación para disminuir los impactos negativos y potenciar los positivos. Y en este caso, son los equipos multidisciplinares los que tienen un gran papel en la búsqueda de estrategias que supongan una transición equilibrada y no un cambio drástico.

\section{BIBLIOGRAFÍA}

1. Schooler C, Mulatu MS. The reciprocal effects of leisure time activities and intellectual functioning in older people: A longitudinal analysis. Psychology and aging 2001; 3: 466-482.

2. Newson RS, Kemps EB. General Lifestyle Activities as a Predictor of Current Cognition and Cognitive Change in Older Adults: A Cross-Sectional and Longitudinal Examination. The Journals of Gerontology Series B: Psychological Sciences and Social Sciences 2005; 60: 113-120.

3. Costa G, Sartori S. Flexible work hours, ageing and well-being. International Congress Series, 1280, 2005, 23-28, Assessment and Promotion of Work Ability, Health and Well-being of Ageing Workers.

4. Seitsamo J, Tuomi K, Martikainen R. Activity, functional capacity and well-being in ageing Finnish workers. Occupational Medicine 2007; 57 (2): 85-91.

5. Phillipson C. The transition to retirement. In: Social change and life course. G. Cohen, (ed.). London: Tavistock Publications, 1987.

6. Marquié, J-C, Paumès D, Volkoff S. Working with age. London: Taylor \& Francis, 1998.

7. Baltes PB. Successful Aging: Perspectives from the Behavioral Sciences. Cambridge University Press, 1993.
8. Raz N, Rodrigue KM. Differential aging of the brain: Patterns, cognitive correlates and modifiers. Neuroscience and Biobehavioral Reviews, 2006; 30: 730-748.

9. Maguire EA, Gadian DG, Johnsrude IS, Good CD, Ashburner J, Frackowiak, RSJ, Frith CD. Navigation-related structural change in the hippocampus of taxi drivers. Proceedings of National Academy of Science U. S. A. 2000; 97: 4398-4403.

10. Salthouse TA, Babcock RL, Skovronek E, Mitchell DRD, Palmon R. Age and experience effects in spatial visualization. Developmental Psychology, 1990; 26: 128-136.

11. Salthouse TA, Mitchell DRD. Effects of age and naturally occurring experience on spatial visualization performance. Developmental Psychology 1990; 26: 845-854.

12. Ansiau D, Marquié JC, Soubelet A, Ramos S. Relationships between cognitive characteristics of the job, age, and cognitive efficiency. International Congress Series 2005; 1280: 43-48.

13. Freund AM, Baltes PB. Life-management strategies of selection, optimization, and compensation: Measurement by self-report and construct validity. Journal of Personality \& Social Psychology 2002; 82: 642-662. 